URLs:

P-screen database

http://flypush.imgen.bcm.tmc.ed u/pscreen

Tiled primer design program http://flypush.imgen.bcm.tmc.ed $\underline{\mathrm{u} / \text { primer }}$

\section{I can name it in three...}

A new strategy that uses $P$ transposable elements to map Drosophila mutations in three mapping rounds is cheaper than single nucleotide polymorphism (SNP) mapping but equally precise.

When it comes to carrying out a forward genetic screen in flies, nothing could be easier than feeding them a sugar-laced mutagen or zapping them with DNA-damaging rays. Unfortunately, the job ahead - turning that incubator full of mutant lines into mapped mutations - is a process that takes time, considerable effort and generally relies on the existence of a scorable marker. And, even then, you will probably still be more than $300 \mathrm{~kb}$ away from the mutation.

Until now, SNPs seemed to be the most promising tools for more efficient and accurate mapping: these molecular markers are present at such high density in the Drosophila genome that they could be used to pinpoint every gene. However, Grace Zhai, Robin Hiesinger and colleagues show that a bank of thousands of publicly available strains (the $P$-screen database), each containing a molecularly mapped insertion of the $P$ transposable element, can provide single nucleotide accuracy at a much lower price than SNP mapping.

In the first two rounds of mapping, the authors crossed flies with an induced mutation to strains that each contain a single $P$-element insertion. The red eye colour that is conferred by the $P$ element allows the various recombinant classes of fly in the F2 generation to be easily categorized. The number of offspring in each recombinant class, combined with the precise molecular location that exists for all $P$ elements, yields a good estimate of where the mutated nucleotide lies: although the first round only maps the mutation to the chromosome arm, the second round takes you to within 0.2 centimorgans (or $\sim 50 \mathrm{~kb}$ ) of the mutation. The third round leads to the point mutation itself either by the failure of the mutation to complement alleles of candidate genes or, as the authors propose, by using an automated mutationdetection system to identify those fragments in the candidate region that carry a mismatch (to facilitate this step the authors used a Tiled primer design program, which is available online).

Mapping strategies that rely on the outcome of meiotic recombination - and most do - have to contend with the obtrusive fact that

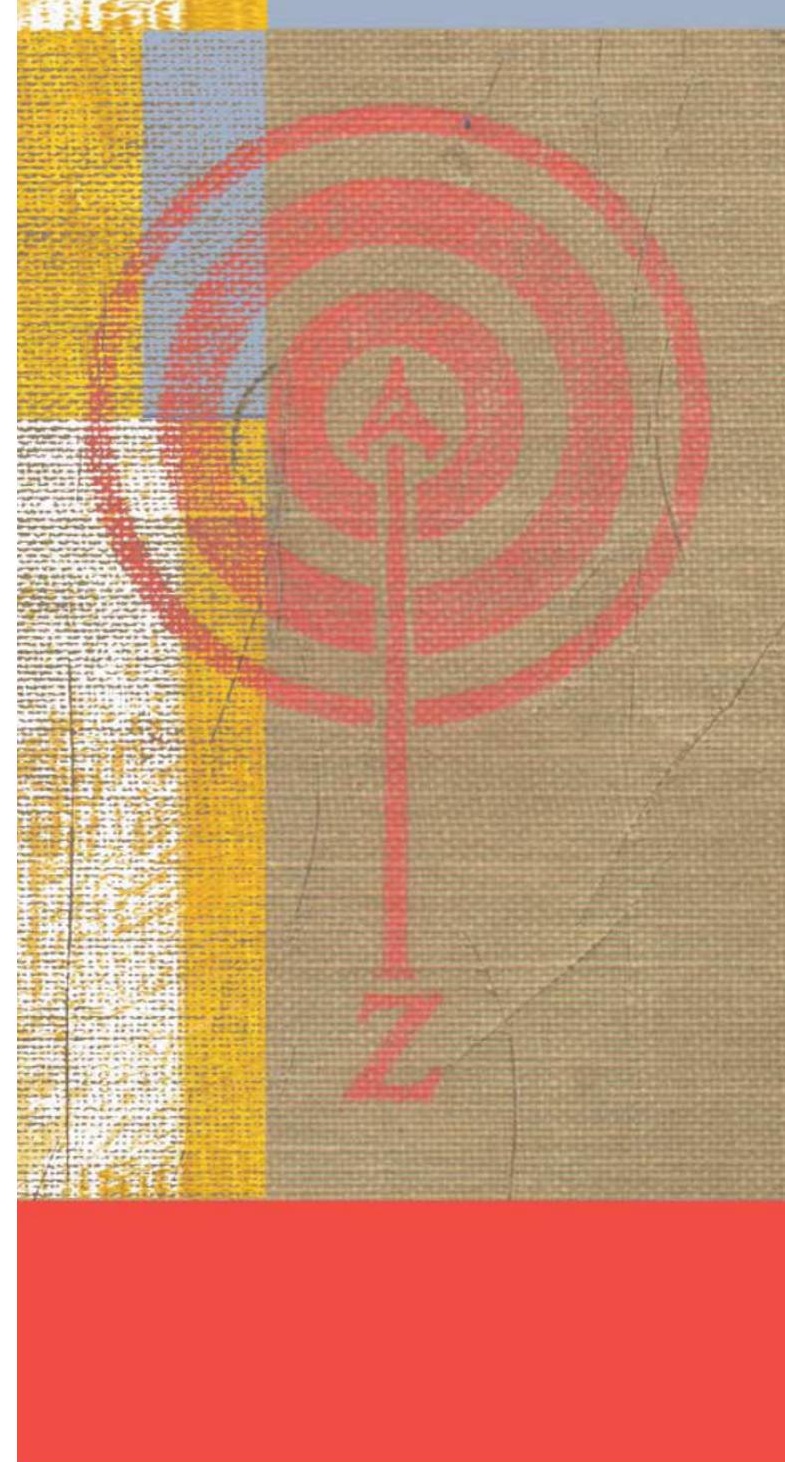

recombination rates (RRs) vary across a chromosome. Variations in RR across the even relatively small interval in which you are trying to locate your mutation could seriously undermine the accuracy of the physical map; indeed, the authors found that in the 15 regions they surveyed the RR could vary by as much as 12 -fold. The paper outlines several routes to overcome serious RR variation - solutions that the authors themselves used to locate 8 of the 15 mutant genes in their study.

This new mapping strategy does not promise to take the pain out of mapping a point mutation, nor does it pretend to be foolproof - for example, further fine-mapping steps are sometimes required. However, it does offer a high throughput, accurate and quick tool that does not break the bank.

Tanita Casci

(1) References and links

ORIGINAL RESEARCH PAPER Zhai, R. G., Hiesinger, P. R. et al. Mapping Drosophila mutations with molecularly defined $P$ element insertions. Proc. Natl Acad. Sci. USA 100, 10860-10865 (2003) WEB SITES

Hugo Bellen's laboratory: http://flypush.imgen.bcm.tmc.edu/lab P-screen database: http://flypush.imgen.bcm.tmc.edu/pscreen Tiled primer design program:

http://flypush.imgen.bcm.tmc.edu/primer 